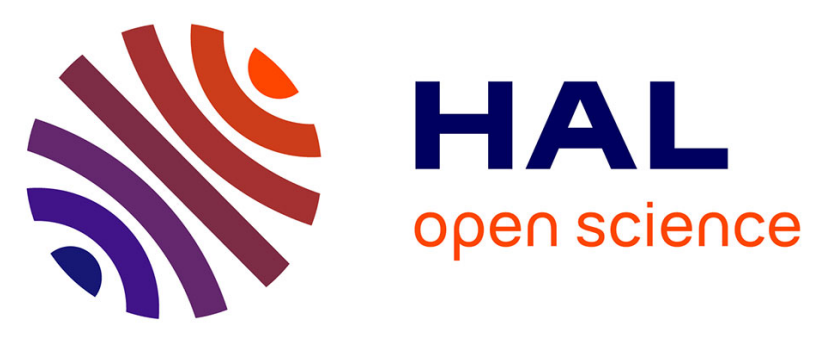

\title{
Decision Making and Strategic Planning for Disaster Preparedness with a Multi-Criteria-Analysis Decision Support System
}

Sascha Schlobinski, Giulio Zuccaro, Martin Scholl, Daniel Meiers, Ralf Denzer, Sergio Guarino, Wolf Engelbach, Kuldar Taveter, Steven Frysinger

\section{To cite this version:}

Sascha Schlobinski, Giulio Zuccaro, Martin Scholl, Daniel Meiers, Ralf Denzer, et al.. Decision Making and Strategic Planning for Disaster Preparedness with a Multi-Criteria-Analysis Decision Support System. 11th International Symposium on Environmental Software Systems (ISESS), Mar 2015, Melbourne, Australia. pp.178-186, 10.1007/978-3-319-15994-2_17. hal-01328547

\section{HAL Id: hal-01328547 https://hal.inria.fr/hal-01328547}

Submitted on 8 Jun 2016

HAL is a multi-disciplinary open access archive for the deposit and dissemination of scientific research documents, whether they are published or not. The documents may come from teaching and research institutions in France or abroad, or from public or private research centers.
L'archive ouverte pluridisciplinaire HAL, est destinée au dépôt et à la diffusion de documents scientifiques de niveau recherche, publiés ou non, émanant des établissements d'enseignement et de recherche français ou étrangers, des laboratoires publics ou privés. 


\title{
Decision Making and Strategic Planning for Disaster Preparedness with a Multi-Criteria-Analysis Decision Support System
}

\author{
Sascha Schlobinski ${ }^{1}$, Giulio Zuccaro ${ }^{3}$, Martin Scholl ${ }^{1}$, Daniel Meiers ${ }^{1}$, Ralf Denzer ${ }^{1,2}$, \\ Sergio Guarino ${ }^{3}$, Wolf Engelbach ${ }^{4}$, Kuldar Taveter ${ }^{5}$, Steven Frysinger ${ }^{1,2}$ \\ cismet GmbH, Saarbrücken, Germany

\begin{abstract}
In the context of the CRISMA FP7 project we have developed a seamless decision support concept to connect simulated crisis scenarios and aggregated performance indicators of impact scenarios with state of the art MultiCriteria Decision Analysis (MCDA) methods. To prove the practicality of the approach we have developed a decision support tool realising the important aspects of the method. The tool is a highly interactive and user-friendly decision support system (DSS) that effectively helps the decision maker and strategic planner to perform multi-criteria ranking of scenarios. The tool is based on state-of-the-art web technologies.
\end{abstract}

Keywords: impact scenario simulation · indicator $\cdot$ criteria $\cdot$ decision support system $\cdot$ multi-criteria-analysis $\cdot$ disaster preparedness $\cdot$ strategic planning

\section{Introduction}

Decision making and strategic planning for disaster preparedness can be extensively supported by simulations of crisis scenarios. Such scenarios can provide insight into the overall course, cause and effect of many aspects of a future crisis. A powerful high level way to look at a crisis is by relating hazards, the exposure of elements at risk and their vulnerabilities, resulting in probabilities of damage or other effects. A simulation following this concept will produce the so-called impact scenarios that show the potential damage of a crisis, e.g. the effect of an earthquake on buildings and population [1]. Impact scenarios are particularly suited to supporting strategic

adfa, p. 1, 2015.

(C) Springer-Verlag Berlin Heidelberg 2015 
decision making as they focus on the causal connection between a specific threat and the potential effects on the objects of concern. The construction of impact scenarios requires the integration of a potentially large number of complex data sets either based on static data (such as census data) or simulation results (such as risk maps). These data usually come from various sources and thus are inherently heterogeneous with respect to format, resolution and semantics.

One way to considerably reduce the complexity of impact scenarios while preserving their key properties is to aggregate scenario data into so called (key) performance indicators [2]. This kind of approach to complex data has a long history in economics and business management e.g. [3] and such indicators are the de-facto standard in measuring the performance of emergency services (e.g. [4]). While performance indicators pertaining to impact scenarios allow comparison of individual indicator values decision makers (DM) still face multiple, often-conflicting decision objectives involving more than one criterion. As a result, the selection of a specific scenario (e.g. intervention) with the "best" performance is very difficult. For example, a particular mitigation action will come with a cost but will reduce the potential impact of a disaster. So the objective of minimal cost for mitigation measures in combination with the objective of minimizing disaster impact leads to a trade-off decision problem (np-hard [5]) where optimization approaches (for example) are hardly applicable in real world solutions. However, established methods of Multi-Criteria Decision Analysis (MCDA) e.g. [6] offer solutions to the problem.

This paper describes a seamless decision support concept developed within the CRISMA FP7 project [7] to connect simulated crisis scenarios and aggregated performance indicators of impact scenarios with state of the art Multi-Criteria Decision Analysis (MCDA) methods [8].

\section{Concept Overview}

The overall idea is to: (a) Let the Decision Maker (DM) produce and use scenarios in support of the decision; (b) provide aggregated but representative information about scenarios (indicators); (c) support the DM in defining an explicit decision strategy (criteria, priorities, Andness and Orness (see section 3)); and (d) assist in comparing and ranking impact scenarios according to the decision strategy. 


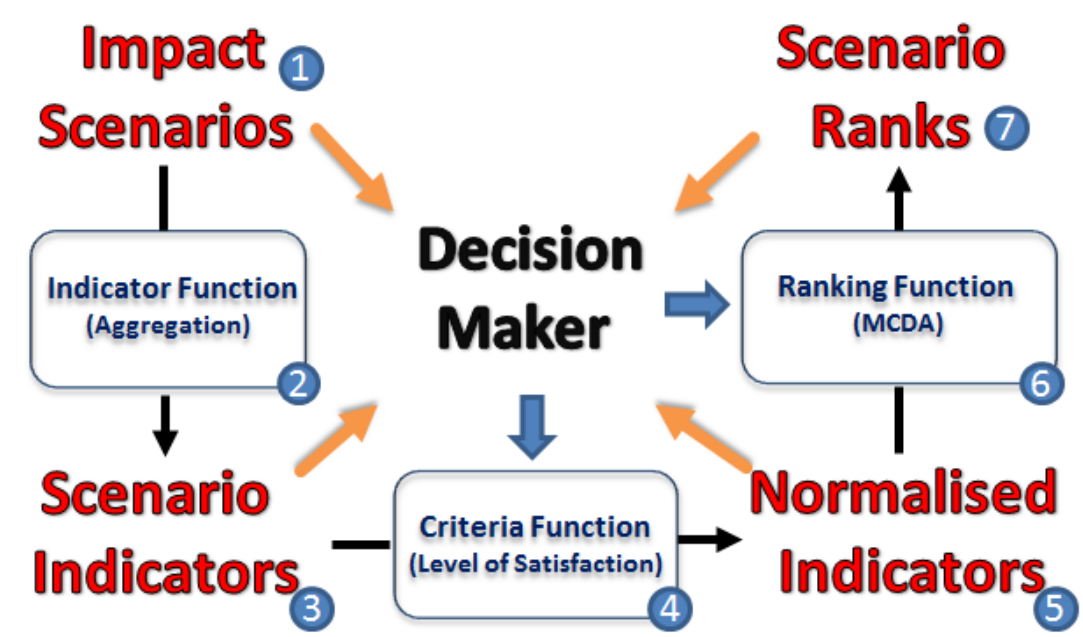

Fig. 1. Decision Support Concept Overview

The overall concept consists of seven elements - four data and three functional - to support the DM: (1) an impact scenario consisting of information required to take a decision, e.g. representing the possible consequences of a flood for people living in the flooded area; (2) an indicator function to map an impact scenario to indicators; (3) a set of representative scenario indicators consisting of aggregated scenario information, e.g. the number of homeless, or the building damage or cost; (4) a criteria function mapping each element of the indicator set to satisfaction; (5) a level of satisfaction in a normalised scale $(0-1$ or $0 \%-100 \%)$; (6) a ranking function mapping normalized indicator sets to values; and (7) corresponding scalar values (ranks/score). The DM can use the four data elements as a basis for the decision and define an individual decision strategy mapping indicators to criteria with the help of criteria functions. In addition they are supported in assigning priorities to indicators as well as defining the level of "Andness" and "Orness" of the ranking function [9] through the parameterization of a MCDA method [6]. More concretely the DM is supported in:

- Using indicators derived from impact scenario data (usually aggregated) to quickly assess and compare impact scenarios

- Defining a decision strategy by:

- Mapping performance indicators to decision criteria (defining the level of satisfaction for each indicator)

- Defining priorities by assigning weights to indicators

- Defining the level of Andness and Orness to be considered when computing the rank of an impact scenario

- Dealing with a multi-criteria decision problem by obtaining a ranking of scenarios with respect to the defined decision strategy. 


\section{Ordered Weighted Averages as a Means for Decision Support}

Decision problems considering more than one criterion on the basis of impact scenarios require appropriate methods to assess the performance of specific scenarios. In our concept we have selected the Ordered Weighted Averages method (OWA) $[6,10,11]$ that allows one to specify a particular decision strategy that defines the properties of a good solution. The OWA method allows us to:

- Implement several decision makers' perspectives (multiple points of view);

- Make the decision strategy explicit;

- Obtain a score/rank for each scenario;

- Let the DM choose between different strategies (e.g. optimistic, neutral, pessimistic);

- Compare results obtained under different strategies.

The OWA method is based on multi-criteria aggregation operators proposed by Yager [6]. OWA is characterized by a vector of ordered weights in addition to the importance weights assigned to each criterion. Using OWA, normalized indicator values are multiplied with a corresponding level of importance. The vector of weighted levels of satisfaction for all indicators is re-ordered according to their values and weighted according to their position in the vector. The vector of ordered weights determines an instance of an OWA operator. E.g. the vector of ordered weights $(1,0, \ldots$, 0 ) will give full weight to the criterion with the highest level of satisfaction independent of all other criteria (maximum level of Orness). As a consequence, alternatives with a single outstanding property will be ranked highest. This is called a risk-taking or optimistic decision strategy. In contrast, the vector of ordered weights $(0, \ldots, 0,1)$ will give full weight to the criterion with the lowest level of satisfaction. As a consequence alternatives with the best "poor" criterion will rank highest (maximum level of Andness). This is called a pessimistic decision strategy. Obviously, between these two extremes there is a large number of intermediate strategies. Another easily interpreted strategy is the neutral strategy that does not emphasize any position in the re-ordered criterion values (simple weighted average). The vector of ordered weights can be calculated to fit to a specific decision strategy [10] but of course they can be defined manually by the DM as will be shown in the next section.

\section{The Implementation}

The concept described above has been implemented including a HTML5 [12] (Angular JS [13]) based User Interface (UI). The software is part of the CRISMA Integrated Crisis Management System Framework [14]. It is available under an open source license as a github project [15]. For illustration, the figures visible in the screenshots 
refer to three fictional alternative mitigation strategies for an earthquake in L'Aquila (Italy), since that is one of case studies addressed in the CRISMA project ${ }^{1}$.

\subsection{Scenario Analysis and Comparison View}

The Scenario Analysis and Comparison View consists of several widgets and visually represents indicator and criteria data to compare different simulated scenarios side by side. The indicators vector is mainly based on quantities (e.g. number of victims who died) calculated from a scenario. To be effectively used in a decision support context indicators need to be qualified. Here qualification basically means assigning a level of satisfaction to the indicator data. The "normalised" indicators can be better used as decision criteria. As indicators and criteria data have the same format (vector of scalar values) both can be displayed in the same fashion.

\subsubsection{Indicator Table Widget}

Fig.2 shows the normalised indicators of three example scenarios in a tabular form.

\begin{tabular}{|c|c|c|c|}
\hline 囲 Criteria Table & & & Change Mode - \\
\hline Criteria & L'Aquila ( $M=7+B R$ ) & L'Aquila (M=7) & L'Aquila (M=7) (edited) \\
\hline \multicolumn{4}{|l|}{ ACasualties } \\
\hline Number of dead & 80 Percent & 90 Percent & 54 Percent \\
\hline Number of homeless & 9 Percent & 38 Percent & 83 Percent \\
\hline Number of injured & 68 Percent & 67 Percent & 79 Percent \\
\hline \multicolumn{4}{|l|}{ \$ Economic cost } \\
\hline Direct damage cost & 76 Percent & 86 Percent & 53 Percent \\
\hline Indirect damage cost & 67 Percent & 70 Percent & 65 Percent \\
\hline Direct restoration cost & 47 Percent & 5 Percent & 65 Percent \\
\hline \multicolumn{4}{|l|}{ 全Damaged buildings } \\
\hline Lost buildings & 73 Percent & 7 Percent & 1 Percent \\
\hline Unsafe buildings & 8 Percent & 97 Percent & 8 Percent \\
\hline \multicolumn{4}{|l|}{ A Damaged Infrastructure } \\
\hline Number of damaged road segments & 30 Percent & 45 Percent & 96 Percent \\
\hline \multicolumn{4}{|l|}{$\Delta$ Evacuation cost } \\
\hline Total evacuationcost & 28 Percent & 45 Percent & 84 Percent \\
\hline
\end{tabular}

Fig. 2. Criteria Table

\footnotetext{
${ }^{1}$ For reasons of security sensitivity the data used in this paper consist of test data sets that do not reflect reality in any way
} 


\subsubsection{Criteria Function Definition Widget}

The Criteria Function Definition Widget depicted in Fig. 3 allows the definition of functions converting indicator values to criteria.

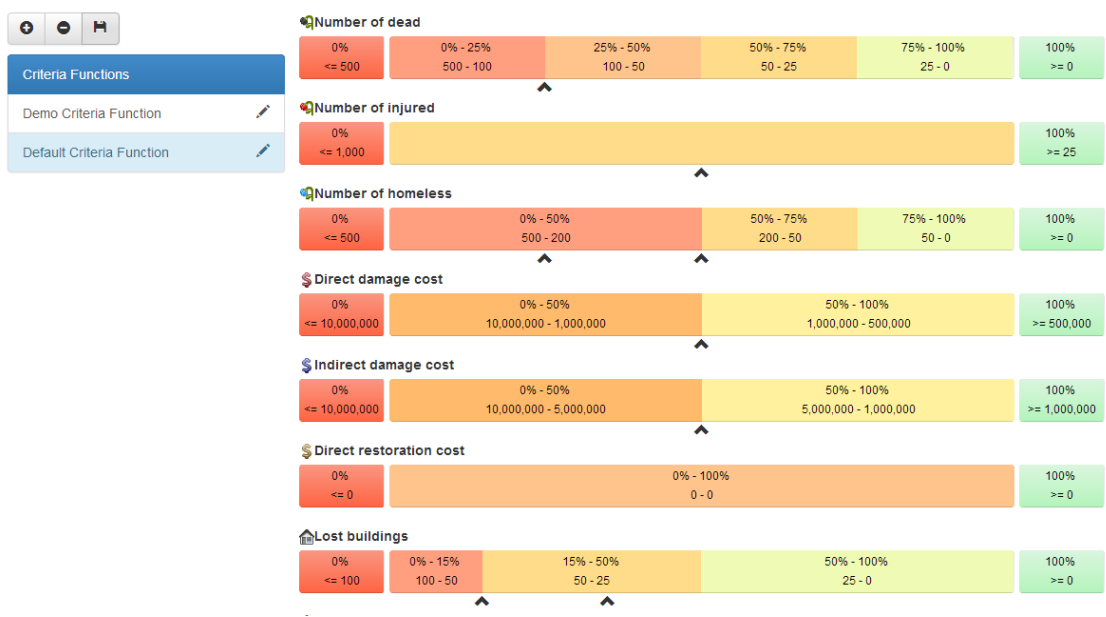

Fig. 3. Criteria Function Definition

The view presented in Fig.4 allows users to correlate individual indicator and criteria values.

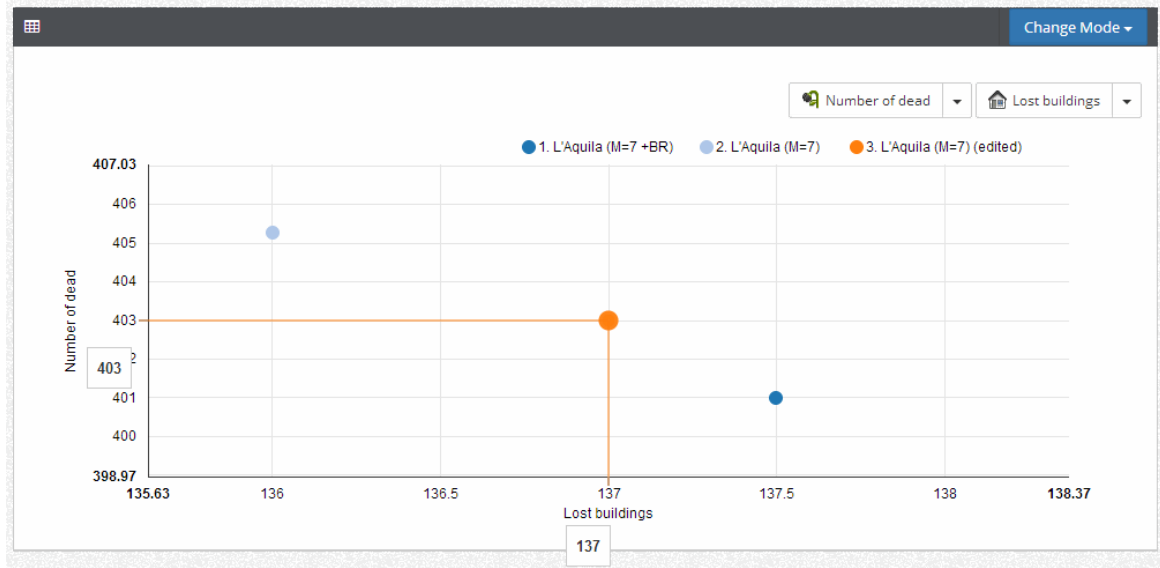

Fig. 4. Indicator Scatter Plot

Fig. 5 shows the data as spider charts in order to support the quick assessment of the overall performance of the selected scenarios. In addition a "reference scenario" (in the example L'Aquila ( $M=7)$ visualised in orange) can be selected. 


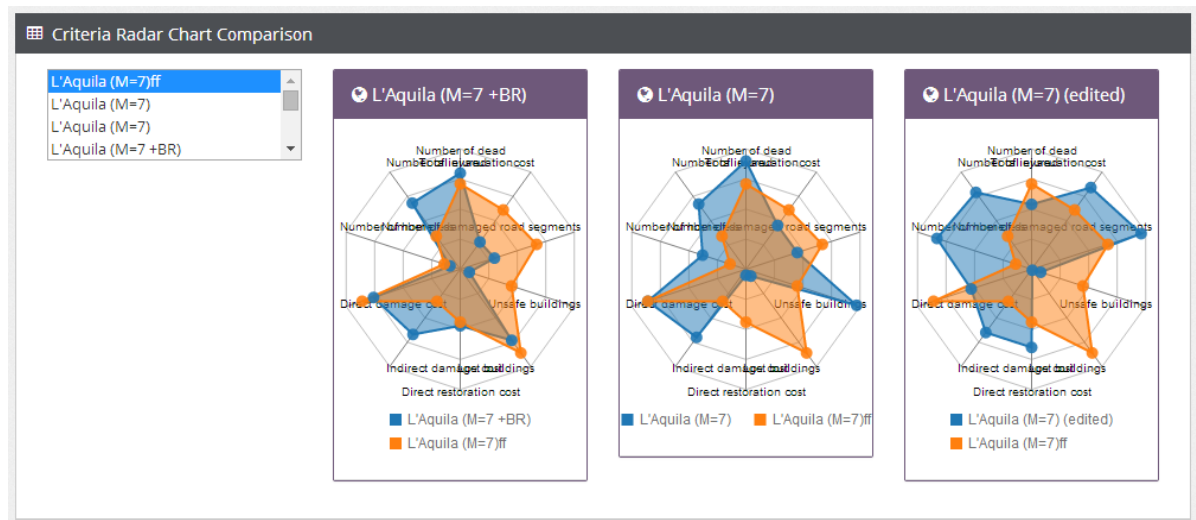

Fig. 5. Criteria Radar Chart

\subsection{Multi Criteria Analysis and Decision Support View}

While the Scenario Analysis and Comparison View (see section 4.1) allows a comparison of indicators and criteria for different scenarios, the Multi Criteria Analysis and Decision Support View allows a ranking of different scenarios with respect to a specific decision strategy.

In this it adds supplemental decision support functionalities. The view is composed of two different widgets: The Decision Strategy Widget and the Decision Ranking Widget.

\subsubsection{Decision Strategy Widget}

The Decision Strategy Widget allows one to define a weighting strategy for different criteria. In this way, a weighting factor can be assigned to each indicator. This factor scales the contribution of the particular criteria to the overall scenario rank. An additional weighting factor can be selected to weigh criteria in relation to the achieved level of satisfaction. This is done according to the OWA method (see section 3). 


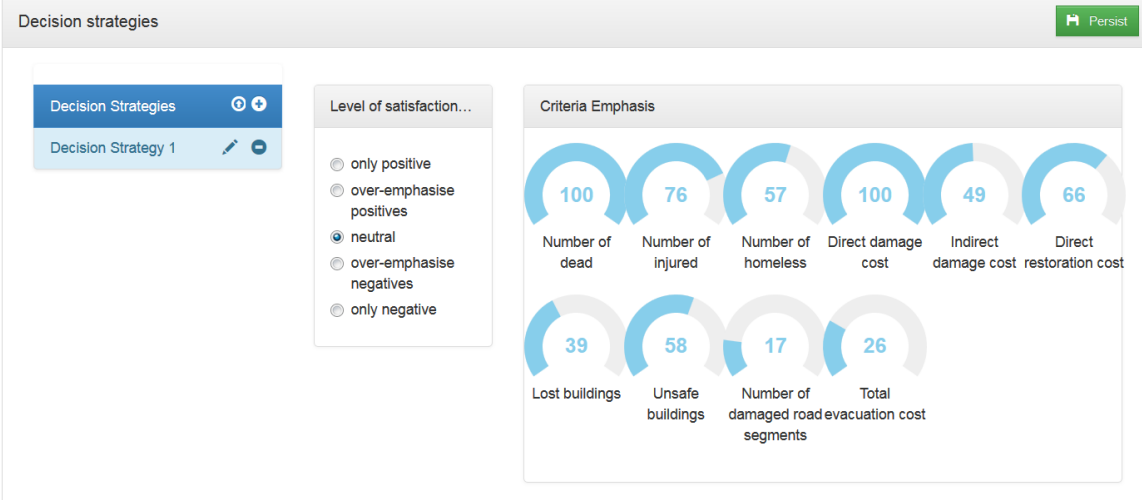

Fig. 6. Decision Strategy Definition

\subsubsection{Decision Ranking Widget}

The Decision Ranking Widget allows the selection of a previously defined decision strategy and criteria function. It applies the selection to the available scenarios and produces a ranking.

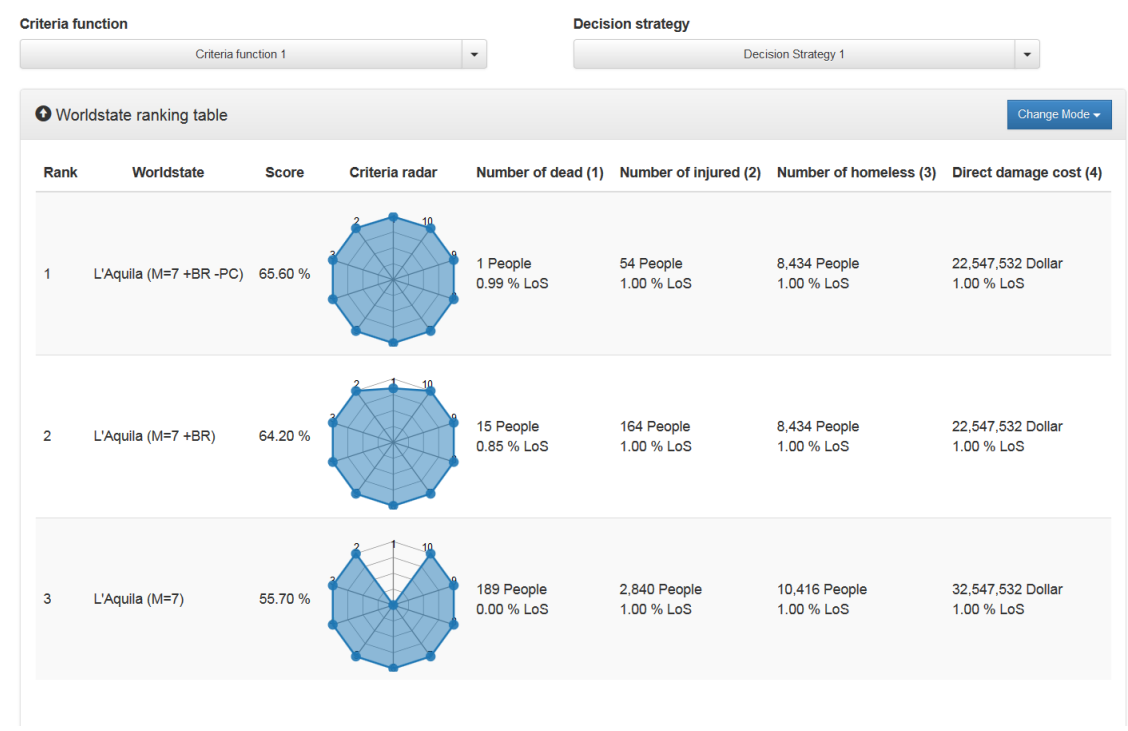

7. Scenario Ranking

Fig.

\section{Conclusion and Outlook}

We have presented a seamless decision support concept to intended connect simulated crisis scenarios and aggregated performance indicators of impact scenarios with state- 
of-the-art Multi-Criteria Decision Analysis (MCDA) methods. To prove the practicality of the approach we have developed a decision support tool realising the important aspects of the method. The tool effectively supports the decision maker and strategic planner in applying a multi-criteria decision strategy to the ranking of scenarios. The tool is based on state-of the art web technologies and is freely available under an open source license. Currently, the presented approach and DSS are evaluated in a number of different disaster management preparedness case studies in the context of the project.

Future work includes the integration of uncertainty indicators that will allow decision makers to take the inherent uncertainty of scenario data into account. Also, as we do not necessarily know in advance what is important for successful crisis management (what are the relevant indicators). Crisis managers may need to explore what has led to particular indicator values rather than what are the values of particular indicators. We plan to work out a method and the corresponding software enabling to compare the dynamics of scenario evolvement in alternative scenarios.

\section{Acknowledgements}

The research leading to this paper has been performed within the CRISMA project, which is co-funded under the European Community's Seventh Framework Programme FP7/2007 - 2013 (grant agreement no. 284552).

\section{References}

1. G. Zuccaro, F. Cacace (2010) Seismic Casualty Evaluation: The Italian Model, an Application to the L'Aquila 2009 Event, Human Casualties in Earthquakes Advances in Natural and Technological Hazards Research Volume 29, 2011, pp 171-184 Date: 08 Dec 2010

2. W. Engelbach, S. Frings, R. Molarius, C. Aubrecht, M. Meriste, A. Perrels: Indicators to compare simulated crisis management strategies. 5th International Disaster and Risk Conference IDRC Davos 2014

3. Business Measures-Modes and Key Performance Indicators http://publib.boulder.ibm.com/infocenter/dmndhelp/v6rxmx/index.jsp?topic=/com.ibm.bto ols.help.modeler.bmeasures.doc/doc/concepts/measures/kpis.html

4. Key Performance Indicators in EMS Zygowicz, W.; http://www.usfa.fema.gov/pdf/efop/efo44984.pdf

5. Garey M.R., Johnson D.S, Computers and Intractability; A Guide to the Theory of NPCompleteness, W. H. Freeman \& Co. New York, NY, USA (C1990 ISBN:0716710455

6. Yager, R.R. 1988. On ordered weighted averaging aggregation operators in multi-criteria decision making. IEEE Transactions on Systems, Man and Cybernetics, 18 (1), 183-190.

7. A.-M. Heikkilä, R. Molarius, T. Rosqvist, A. Perrels, Minimising the outcome of disasters by simulating the effects of different actions. The Second Nordic International Conference on Climate Change Adaptation. Helsinki, 29-31 Aug. 2012

8. Steuer, R.E. (1986). Multiple Criteria Optimization: Theory, Computation and Application. New York: John Wiley 
9. Dujmović, J. 2007 Properties of Local Andness/Orness. Theoretical Advances and Applications of Fuzzy Logic and Soft Computing

Advances in Soft Computing Volume 42, 2007, pp 54-63

10. Yager, R.R. 1996.Quantifier guided aggregation using OWA operators. International Journal of Intelligent Systems, pp. 11-49.

11. Zuccaro, G. \& Filomena, P. 1997. Multi-criteria analysis in vulnerability assessment. Second Egyptian Conference on Earthquake Engineering, Aswan, Egypt.

12. http://www.w3.org/TR/html5/

13. https://angularjs.org/

14. P. Dihé, R Denzer, M. Polese, A. Heikkilä, D. Havlik, J. Sautter, Th Hell, S. Schlobinski, G. Zuccaro, W. Engelbach (2013), An architecture for integrated crisis management simu lation 20th International Congress on Modelling and Simulation (MODSIM2013); Adelaide, South Australia, Sunday 1 to Friday 6 December 2013

15. https://github.com/crismaproject 